\title{
Les mutations de la filière viticole en France
}

Des vignerons de plus en plus engagés dans des pratiques durables:

l'exemple alsacien

Mutations in the wine industry in France

Sylvaine Fassier-Boulanger

\section{(2) OpenEdition \\ Journals}

Electronic version

URL: https://journals.openedition.org/rge/5165

DOI: $10.4000 /$ rge. 5165

ISSN: 2108-6478

Publisher

Association des géographes de l'Est

Printed version

Date of publication: 15 October 2014

ISSN: 0035-3213

Electronic reference

Sylvaine Fassier-Boulanger, "Les mutations de la filière viticole en France", Revue Géographique de l'Est [Online], vol. 54 / 1-2 | 2014, Online since 18 October 2014, connection on 07 December 2022. URL: http://journals.openedition.org/rge/5165 ; DOI: https://doi.org/10.4000/rge.5165

This text was automatically generated on 29 September 2020 .

All rights reserved 


\section{Les mutations de la filière viticole en France}

Des vignerons de plus en plus engagés dans des pratiques durables : l'exemple alsacien

Mutations in the wine industry in France

Sylvaine Fassier-Boulanger

1 La filière viticole affiche un chiffre d'affaires de 12 milliards d'euros ${ }^{1}$, fournit plus de 600000 emplois directs et indirects en France et représente plus de 15\% de la valeur de la production agricole. La production varie entre 43 et 50 millions d'hectolitres selon les années (2008-2012), soit environ 17\% des volumes mondiaux produits. Pourtant, le marché du vin a connu de profonds bouleversements entre 1971-1973 et 2008-2010 avec une progression de $10 \%$ des rendements viticoles moyens tandis que les superficies mondiales ont diminué de $24 \%$ et la consommation de $12 \%$ (Peynaud, Blouin, 2012). «L'évolution française est encore plus violente : surface (- $36 \%)$, volume $(-33 \%)$, consommation nationale $(-48 \%)$ mais exportations de $+125 \%$, soit $36 \%$ de sa production (contre $9 \%$ ) et $16 \%$ des exportations mondiales (contre $15 \%$ ). Dans le même temps, on est passé de plus d'un million de producteurs à moins de cent quarante mille » (Peynaud, Blouin, 2012).

2 Pour continuer de s'affirmer sur la scène française et internationale et répondre aux demandes de la société, la filière continue de s'adapter et de réformer ses structures, avec difficultés parfois, eu égard au poids des traditions dans les pratiques agricoles. Depuis les années 1980, le système productif vitivinicole français tend vers un lent développement de la durabilité des pratiques de culture et de vinification. Certaines régions viticoles, telle l'Alsace, se sont engagées dans cette voie en précurseurs.

3 En quoi consistent les pratiques durables que les vignerons tentent de développer dans les exploitations françaises depuis les années 1980 ? Pour quelles raisons et de quelles façons s'imposent-elles progressivement dans le vignoble français et plus particulièrement en Alsace? 
4 Nous proposerons des éléments de réponses à ces interrogations en nous penchant exclusivement sur l'un des acteurs de cette filière : le vigneron. Nous montrerons tout d'abord que la préoccupation première de celui-ci durant les Trente Glorieuses est de produire avant tout, en soulignant les pratiques qui ont cours avant l'apparition de la notion de viticulture durable. Puis, nous insisterons sur les évolutions du secteur rendues nécessaires depuis les années 1980-1990 en insistant sur l'affirmation de la durabilité. Enfin, nous montrerons concrètement la façon dont l'Alsace s'est engagée dans cette voie à partir d'une enquête menée en 2011.

\section{Un vignoble dont la modernisation s'inscrit dans la modernisation agricole globale des Trente Glorieuses}

\section{A. Une utilisation accrue d'intrants chimiques pour assurer une production régulière et de bonne qualité sanitaire}

5 Le système productif, encouragé par la Politique Agricole Commune et permis par l'essor de l'industrie chimique, développe l'usage d'engrais et de produits de traitement (pesticides, herbicides, fongicides) dans les vignes afin de garantir la récolte: les fongicides tuent les champignons responsables de maladie tel le mildiou ${ }^{2}$, les insecticides éradiquent les insectes vecteurs de maladies ou de dégâts comme les cicadelles (flavescence dorée) et les herbicides détruisent les mauvaises herbes qui concurrencent la vigne et entretiennent parfois trop d'humidité. Le nombre de traitements réalisés sur chaque parcelle de vigne est variable (de 8 à 18 par an) et dépend de la localisation géographique de chaque vignoble. Ces traitements, très bien accueillis par les vignerons, sont encouragés par l'essor de la mécanisation : le tracteur enjambeur, tracteur haut et étroit pouvant passer dans les rangs de vigne, facilite le rognage ${ }^{3}$, la multiplication des pulvérisations donc des traitements chimiques, et développe les vendanges mécaniques dans certains vignobles.

6 Le travail du sol entre les rangs de vigne pour limiter la pousse des adventices est progressivement délaissé au profit des herbicides pulvérisés. Le gain de temps et la moindre fatigue sont appréciés par les vignerons qui sont encore largement des polyculteurs au cours des décennies 1950-70.

7 Les années 1950 voient la vinification mieux maîtrisée par les vignerons, de même que l'essor des coopératives (Legouy, 2008). Les Appellations d'Origine participent aussi largement à la modernisation des vignobles dans la mesure où elles imposent l'usage de cépages de qualité.

\section{B. Un essor des cépages de qualité en lien avec le développement des AOC}

8 Après les ravages causés par le phylloxera ${ }^{4}$ principalement au cours des années 1863-1910, le vignoble français se reconstitue intégralement. Cependant, les vignerons souvent ruinés replantent des producteurs directs ${ }^{5}$ de nouveau touchés par le phylloxera. Le salut vient du greffage de cépages de qualité sur des pieds américains insensibles à l'insecte, technique que les vignerons doivent entièrement apprendre. Avec l'apparition (1936) puis le développement des Appellations d'Origine Contrôlée 
viticoles l'encépagement de qualité se développe : tout vin candidat à l'AOC doit respecter un cahier des charges qui impose au vigneron plusieurs cépages traditionnellement cultivés dans une région donnée et produisant des vins spécifiques et de qualité. L'augmentation des demandes d'Appellation après la deuxième guerre mondiale va donc de pair avec l'arrachage des cépages très productifs au profit de cépages aux rendements moins élevés, garants d'une meilleure qualité de vin (Legouy, 2014). Cette évolution vers la qualité est très progressive et certains vignobles, comme le vignoble languedocien, continuent à utiliser massivement jusqu'à la fin des années 1980 la dénomination de vins de pays (ou vins de table) afin d'écouler les abondantes quantités de vin produites par les cépages carignan ou aramon.

\section{Des évolutions du secteur vitivinicole rendues nécessaires depuis 1980-1990}

\section{A. La filière doit s'adapter à un contexte global de diminution de la consommation de vin en France et de concurrence des vins étrangers}

9 La France étant le principal marché des vins français, l'évolution de sa consommation (Legouy, 2008) est un facteur primordial de l'avenir de la filière. La consommation vinicole française, tous vins confondus (rouges, blancs, rosés/ vins tranquilles ou effervescents), baisse régulièrement depuis 30 ans, passant de 100 litres par habitant par an en 1975 à moins de 50 litres en 2010 (France Agrimer, 2010) ${ }^{6}$. Par ailleurs, le vin est essentiellement consommé lors d'événements particuliers ou est associé à la fête. Les consommateurs réguliers représentent 17\% des Français en 2010, tandis que 45\% d'entre eux sont des consommateurs occasionnels, $38 \%$ ne consommant pas de vin. La filière doit donc s'adapter à la demande occasionnelle en plein essor depuis 1980, dans un contexte de concurrence plus vive avec la rapide ascension des vignobles d'Australie, d'Argentine, du Chili ou d'Afrique du Sud qui n'ont pas à assumer les mêmes contraintes réglementaires qu'en France: plantation de ceps sans droits de plantation, choix de cépages et de savoir-faire dans les pratiques culturales et de vinification, libre irrigation...

10 La production mondiale de vins tente de s'ajuster à la demande en limitant la surproduction avérée depuis 2007-2008, et en produisant de la qualité. En Europe, les superficies diminuent grâce aux primes à l'arrachage de la PAC, notamment chez les trois plus grands producteurs mondiaux : France, Italie et Espagne. Pour faire face à la concurrence viticole mondiale, l'UE met aussi en place en 2008 la nouvelle Organisation Commune du Marché vitivinicole. Deux catégories de vins ont désormais cours :

11 - les vins liés à une origine géographique protégée : les Appellations d'origine protégée (AOP), issues des AOC, et les Indications géographiques protégées (IGP).

12 - les vins sans indication géographique, avec ou non, la mention du cépage et/ou du millésime. La possibilité d'indiquer une mention valorisante (le nom du cépage et /ou l'année) pour un vin sans indication géographique révolutionne la segmentation traditionnelle française et européenne et encourage la compétitivité de la filière sur ce segment de produit où la concurrence internationale est vive. Jusqu'à cette date, les 
vins français affichaient essentiellement le lieu-dit de fabrication pour souligner le rôle du terroir, tandis que l'Alsace faisait figure d'exception avec des vins dits de cépages.

Le système de production vinicole est fondé sur l'origine et la qualité (90\% de l'offre française). Ces deux points sont à la fois des forces et des faiblesses: des forces en termes de qualité et de revenus élevés possibles, des faiblesses liées à la réglementation qui entrave l'innovation et à la notion de terroir difficile à appréhender dans le monde anglo-saxon. Plus encore, le système officiel des AOC et l'inflation de leur nombre ont rendu moins lisible l'univers du vin auprès des consommateurs. A partir des années 1980, la filière viticole française doit s'adapter pour continuer à vendre ses vins en France et à l'étranger.

Sur le plan des structures et de la modernisation des vignobles, l'encépagement s'est considérablement amélioré depuis 1980 sous l'effet de l'arrachage définitif soutenu par les primes de l'UE. Les surfaces plantées en cinsault et en aramon ont diminué, tandis que celles en syrah ou en merlot ont augmenté pour répondre à la demande mondiale de vins couramment dénommés "vins de cépage». Le vignoble languedocien, qui couvrait 407300 hectares en 1979, est passé à 236500 ha en 2010, soit une baisse de $42 \%$ entre les deux dates, avec une accélération entre 2000 et 2010. Le carignan qui représentait 43\% de l'encépagement régional en 1979, n'en représente plus que $13.5 \%$. Mais les efforts restent à poursuivre dans ce sens, le carignan étant considéré comme un cépage inadapté à la demande mondiale ${ }^{7}$. Signe d'une évolution profonde vers la qualité et d'une volonté d'afficher des vins de cépages sur le marché, la syrah, qui était peu fréquente dans cette région en 1979, est devenue le premier cépage en superficie avec un peu plus de 40000 hectares en 2010.

Pourtant, les crises sanitaires et alimentaires des années 1990 ont un retentissement indirect sur la filière vitivinicole.

\section{B. Une volonté renouvelée de lien au terroir dans un contexte de crises alimentaires}

16 La filière viticole doit «se placer dans une perspective d'expansion dynamique et ambitieuse pour que la viticulture reste un secteur stratégique pour la France en matière d'emploi, d'animation des territoires, d'équilibre de la balance commerciale et de relance de l'activité économique » (France Agrimer, 2014), tout en tenant compte de la demande sociétale en termes de traçabilité et de qualité des produits, du changement climatique qui peut avoir des impacts « potentiels forts sur la production (typicité des produits, nouveaux cépages...), sur les conduites de vignobles » (France Agrimer, 2014), et ce, dans un contexte réglementaire international dans lequel il est nécessaire que la France et l'Europe défendent leurs produits. La filière viticole doit donc proposer un produit sain lorsqu'il est consommé raisonnablement, et élaboré en limitant les atteintes sur l'environnement. Le respect du terroir, c'est-à-dire celui des composantes naturelles de la parcelle mises en valeur par un savoir-faire ancestral qui n'altère ni le support ni le fruit, est une demande forte de la société.

17 En effet, le système productif encouragé par la PAC incite à une forte utilisation d'engrais et de produits phytosanitaires. Les impacts de ces produits sur le substrat (destruction de la microfaune dans les couches superficielles du sol), l'air (pulvérisations) et les eaux (eaux de ruissellement chargées de produits chimiques, eaux souterraines polluées par infiltration), de même que sur la santé de l'exploitant 
sont négligés jusqu'aux années 1980. La question des résidus de ces intrants dans le sol ou dans le produit alimentaire et, in fine, pour le consommateur, ne préoccupe guère, ni la population, ni les pouvoirs publics, tant il faut assurer l'autosuffisance alimentaire. Ce n'est qu'en 1991 que la Mutualité Sociale Agricole (la sécurité sociale des viticulteurs) met en place un réseau de toxico-vigilance qui recense les accidents liés à l'emploi des pesticides: allergies, eczémas, troubles hépato-digestifs, respiratoires... Or, les scandales alimentaires du début des années 1990 bouleversent le système agricole et placent au premier plan le rapport entre les pratiques culturales et la santé alimentaire. Les crises de la vache folle en 1992 en France, de la listéria (1993), du poulet et des œufs à la dioxine (1999)... ébranlent certains pans du système productif. Une angoisse diffuse liée à la qualité de l'alimentation apparaît : l'agriculteur peut empoisonner le consommateur, même indirectement. Le lien de confiance qui existait de la fourche à la fourchette est rompu, comme le souligne Claude Fischler qui éclaire les rapports que les Français entretiennent avec leur alimentation (Fischler, Masson, 2008).

Le vin subit de façon indirecte les effets de ces crises par une demande renouvelée du consommateur d'un fort lien du vin au terroir. Cette volonté de se rapprocher du terroir passe d'abord par la demande d'un meilleur respect de celui-ci. Le consommateur est conscient de l'impact des pratiques agricoles sur l'environnement : érosion et épuisement des sols, forts prélèvements de la ressource en eau, abus des traitements phytosanitaires... Or, le terroir symbolise le patrimoine, le monde rural rassurant, et le consommateur tient à cette image même s'il méconnaît le monde rural (Fischler, 2000). Par ailleurs, la société exige de l'agriculteur d'afficher des paysages soignés, de protéger la biodiversité, tant en proposant des produits savoureux et sains. «Les agriculteurs sont à la fois gardiens d'un patrimoine alimentaire, gastronomique, culinaire et en même temps médiateurs des transformations de l'agriculture moderne » (Poulain, 2008).

19 La réduction de l'utilisation des produits phytosanitaires est l'une des composantes essentielles des objectifs de durabilité des pratiques agricoles (plan Ecophyto 2018 et Grenelle de l'Environnement). En France, les grandes cultures représentent 75 \% de l'utilisation des produits phytosanitaires en termes de dépenses, la viticulture $15 \%$, l'arboriculture $5 \%$ et le maraîchage également $5 \%$ (Agreste, 2010). Par contre, par unité de surface, les utilisations les plus importantes sont le fait de la viticulture, de l'arboriculture et du maraîchage et, en grandes cultures, des pommes de terre. Les vignerons sont donc principalement concernés par les mesures européennes qui interdisent certaines substances. La directive communautaire 67/458 distingue les substances chimiques cancérigènes, mutagènes et reprotoxiques (qui altèrent la fertilité ou la reproduction).

20 L'agriculture biologique apparaît souvent, pour le grand public, comme une réponse évidente à la demande sociétale d'une plus grande prise en compte de l'environnement dans les pratiques culturales et de la santé du consommateur.

\section{Une viticulture durable qui s'impose très progressivement sous des formes variées}

21 Officiellement reconnue en France en 1981, l'agriculture biologique proscrit toute utilisation de molécule chimique de synthèse. Elle semble être la panacée face aux 
excès du productivisme. Pourtant, rares sont les vignerons à s'engager dans cette voie au cours des années 1980 et ce, pour différentes raisons: pour vendre son vin sous le label $\mathrm{AB}$, l'exploitant doit s'engager dans une conversion de trois ans de ses parcelles, période pendant laquelle ses rendements diminuent d'au moins $30 \%$. Par ailleurs, les méthodes de culture biologiques sont encore méconnues, même des conseillers viticoles des chambres d'agriculture. Les tâtonnements sont nombreux, et le demeurent encore parfois aujourd'hui.

Ce sont les scandales alimentaires, les problèmes de santé des vignerons et les aides européennes à la conversion «au bio » qui donnent un réel élan à la viticulture biologique.

L'agriculture biologique n'est rentable que sur le long terme lorsque le sol retrouve une certaine fertilité par une plus grande richesse en micro-organismes. Surtout, elle ne constitue qu'une réponse parmi d'autres à la demande de la société en termes d'environnement et de santé. D'autres pratiques répondent à certains objectifs de l'agriculture durable. Celle-ci est l'application à l'agriculture des principes du développement durable ${ }^{8}$ (Schéma 1). Elle se définit par «une production agricole économiquement viable, socialement équitable, et qui ne nuit ni à l'environnement ni à la santé $»^{9}$. Elle comporte trois dimensions principales :

- une dimension écologique : les atteintes sur l'environnement doivent être limitées ;

- une dimension économique : l'exploitation agricole doit être rentable économiquement ;

- une dimension sociale : le bien-être de l'exploitant est pris en compte.

Schéma 1 : La nécessité d'une agriculture durable s'impose à la société à la suite des abus du productivisme agricole

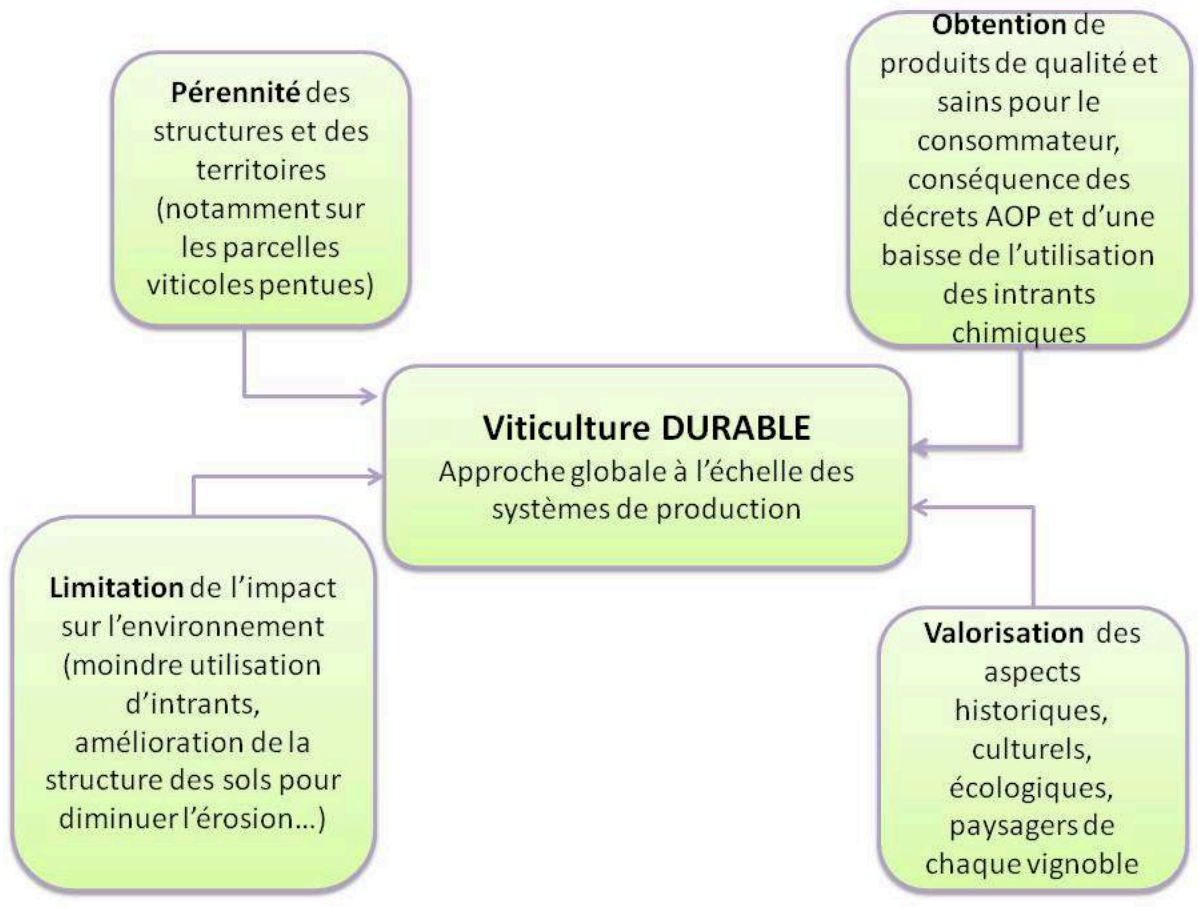

Réalisation : S.Boulanger.

Le premier et principal chemin vers la durabilité est la lutte raisonnée (FassierBoulanger, 2008). Cette dernière s'est progressivement étendue chez les vignerons 
depuis le milieu des années 1990 même si l'expression même de lutte raisonnée a régulièrement été dénigrée par les producteurs car elle tendait à souligner le manque de réflexion de leur part. L'agriculture raisonnée est essentiellement fondée sur la diminution des quantités d'intrants. Les viticulteurs sont soutenus dans cette démarche par de nombreux acteurs: conseillers des chambres d'agriculture, syndicats d'appellation, institut technique de la vigne et du vin... Les bulletins d'alerte météorologique, parfois établis à la parcelle près constituent une aide indispensable : les jours d'application des traitements et les doses préconisées sont déterminés en fonction du risque local de pluie, de forte chaleur... La prévention est privilégiée. Parallèlement, les réglementations européenne puis française ${ }^{10}$ ont interdit l'usage de produits dont la nocivité était trop forte, tel l'arsénite de soude en 2001, en raison de ses effets cancérigènes et toxiques sur la santé. Ce produit était jusqu'alors utilisé en traitement d'hiver pour lutter contre l'esca, maladie du bois qui provoque le dépérissement voire la mort du cep. Pourtant, ces interdictions réglementaires ne sont pas toujours admises par les vignerons qui voient leurs parcelles dépérir et font intervenir leurs élus, comme Roland Courteau, sénateur de l'Aude, qui demande, en vain, au ministre de l'agriculture la possibilité de réutiliser l'arsénite en $2005^{11}$. Depuis 2001, seules les méthodes prophylactiques, d'une efficacité fort variable, sont autorisées: élimination et brûlage les souches mortes porteuses du champignon, limitation des plaies lors de la taille...

La viticulture durable réside aussi dans l'interdiction du nettoyage des pulvérisateurs ou des cuves en plein champ ou dans les rues, afin de limiter les effluents (ou eaux polluées) dans le sol, les cours d'eau ou le tout à l'égout. Des fascicules didactiques établis par l'Institut français de la vigne et du vin sont d'ailleurs diffusés auprès des vignerons afin de les encourager dans ces pratiques.

Afin d'illustrer le passage de la viticulture conventionnelle ${ }^{12}$ à des formes de viticulture durable, nous allons nous pencher sur la viticulture alsacienne, très active en termes de durabilité viticole depuis plus de quinze ans (Fassier-Boulanger, 2006). Une enquête a été menée en septembre 2011 auprès des vignerons alsaciens pratiquant la viticulture biologique et adhérant à la charte des "vins bio " d'Alsace (Vinabio) alors que la législation européenne n'a reconnu ce type de production qu'en $2012^{13}$.

\section{La vitiviniculture durable en Alsace : l'exemple des vignerons respectant la charte Vinabio}

\section{A. Le vignoble alsacien : un vignoble fortement engagé dans l'agriculture biologique}

Plus de $8 \%$ des superficies viticoles françaises sont cultivées en bio en 2013, contre $6 \%$ en 2009. En Alsace, les vignes représentent en moyenne les trois-quarts des surfaces des exploitations alsaciennes en agriculture biologique, ce qui est un cas unique en France. Plus de $13 \%{ }^{14} \mathrm{du}$ vignoble alsacien est biologique en termes de superficie. 


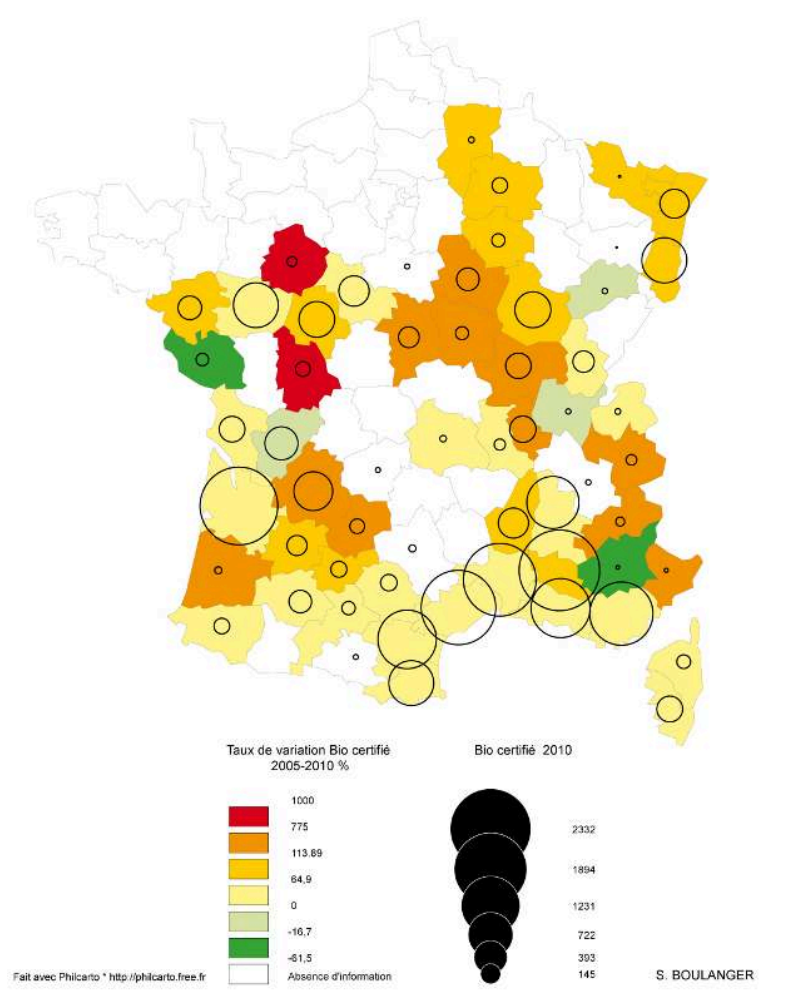

Source : Agence Bio, 2010. Réalisation : F.Legouy et S.Boulanger.

En 2011, année de notre enquête en Alsace, les surfaces viticoles bio (certifiées et en cours de conversion) atteignaient $7,4 \%$ du vignoble national ${ }^{15}$, soit plus de 60000 hectares pour 4700 producteurs environ (1287 en 2001). Les superficies ont gagné $21 \%$ entre 2010 et 2011.

Plus des $2 / 3$ de ces surfaces bio viticoles se situent dans trois régions (Carte 1 ): Languedoc-Roussillon (16 462 ha), Provence-Alpes-Côte-d'Azur (11 209 ha), Aquitaine (7 $715 \mathrm{ha}$ ). Concernant la part des vignes bio ou en conversion dans la surface totale de chaque vignoble, la région PACA arrive en tête (plus de 12\%), suivie de l'Alsace (plus de $11 \%)$, de la Franche-Comté (11\%), de la Corse (plus de $8 \%$ ), de la région Rhône-Alpes (8\%), du Languedoc (8\%)... Le Bordelais reste à la traîne (moins de 5\% de vignes bio en Gironde), la Champagne aussi. Ou bien la renommée n'incite pas à la conversion, ou bien, les vignerons font-ils du bio sans le déclarer.

A la période correspondant à l'enquête, $12 \%$ du vignoble alsacien sont conduits en mode biologique, soit l'équivalent de 255 exploitations viticoles. Ce pourcentage fait de l'Alsace l'une des premières régions de production de vins issus de raisins biologiques en termes de surfaces par rapport aux surfaces totales. Parmi ces exploitations, 38 se sont engagées dans une démarche de qualité et d'identité des vins: ce sont les vignerons adhérant à la Charte Vin Bio d'Alsace avec le logo « Vinabio Alsace ${ }^{16}$ » (15\% des vignerons bio de la région). Cette marque rassemble les producteurs autour d'une démarche commune restrictive pour tendre vers une utilisation minimale de produits œnologiques. Leur volonté est de contrôler le respect du raisin jusque dans la vinification, alors que l'élaboration du vin ne jouit encore d'aucun label en 2011, ni en France, ni en Europe ${ }^{17}$. Ces vignerons, agacés de ne pas voir aboutir de réglementation européenne sur la vinification des vins bio se sont regroupés pour respecter la même 
logique de travail que celle pratiquée dans les vignes et limiter les dérives liées à l'ajout d'additifs lors des vinifications. Ils se conforment, certes, à un cahier des charges de viticulture biologique, mais ajoutent des mesures plus drastiques au cours de la vinification: récolte manuelle afin de préserver l'intégrité des fruits et limiter le tassement des sols, utilisation de soufre limitée à $120 \mathrm{mg} / \mathrm{l}$ pour les vins blancs secs, soit la moitié des normes en cours... Les vignerons adhérant à la charte Vinabio se répartissent dans 24 communes, la grande majorité d'entre eux se situant dans le HautRhin, zone la plus réputée du vignoble alsacien (Ribeauvillé, Kaysersberg,...).

Tout d'abord, un questionnaire a été envoyé à toutes les exploitations adhérant à la charte Vinabio, puis un entretien libre a eu lieu avec les 26 vignerons qui avaient répondu au questionnaire dans le but unique d'affiner leurs réponses. L'enquête avait pour finalité principale de répondre aux questions suivantes : pourquoi des vignerons sont-ils motivés par la viticulture biologique et plus, par une vinification biologique, alors que le label n'existe pas? Quelles sont les caractéristiques de ces vignerons (sexe, âge, type d'exploitation...) ? Mettent-ils en avant leur engagement auprès de leurs voisins, de leurs clients et, dans l'affirmative, comment procèdent-ils?

Un questionnaire (Annexe 1) comportant 55 questions articulées autour de l'histoire de l'exploitation et de l'installation de l'exploitant, de son parcours en faveur de la durabilité des terroirs et de ses actions de valorisation des vins bio, a été envoyé aux adhérents de la Charte Vin Bio d'Alsace. $68 \%{ }^{18}$ d'entre eux ont répondu et l'analyse proposée se fonde sur leurs réponses, augmentées de celles obtenues de divers entretiens afin de compléter leurs retours.

Les motivations des vignerons engagés dans la Charte Vinabio s'inscrivent, de façon intrinsèquement liée, dans des désirs sociétaux et des motivations personnelles multiples.

\section{B. Repenser concrètement les modes de production : prendre soin de l'environnement après des décennies de viticulture conventionnelle}

D'une façon générale, les paysages viticoles alsaciens laissent apparaître de plus en plus de vignes enherbées, avec liserons, coquelicots et pissenlits. La viticulture conventionnelle évolue dans ses pratiques en faveur de la durabilité des terroirs: enherbement ${ }^{19}$ (Photographie 1) et limitation des intrants. L'enherbement, temporaire ou permanent, implanté dans tous les rangs ou dans un rang sur deux, a pour principales vertus d'améliorer la structure et la portance du sol, de le protéger de l'érosion hydrique et éolienne, tout en favorisant le développement de l'activité biologique et la création d'humus. Le vignoble alsacien s'est engagé dans ce nouveau système depuis une décennie. 
Photographie 1 : Une pratique largement développée en Alsace depuis 15 ans : l'enherbement un rang sur deux afin de limiter l'érosion par ruissellement

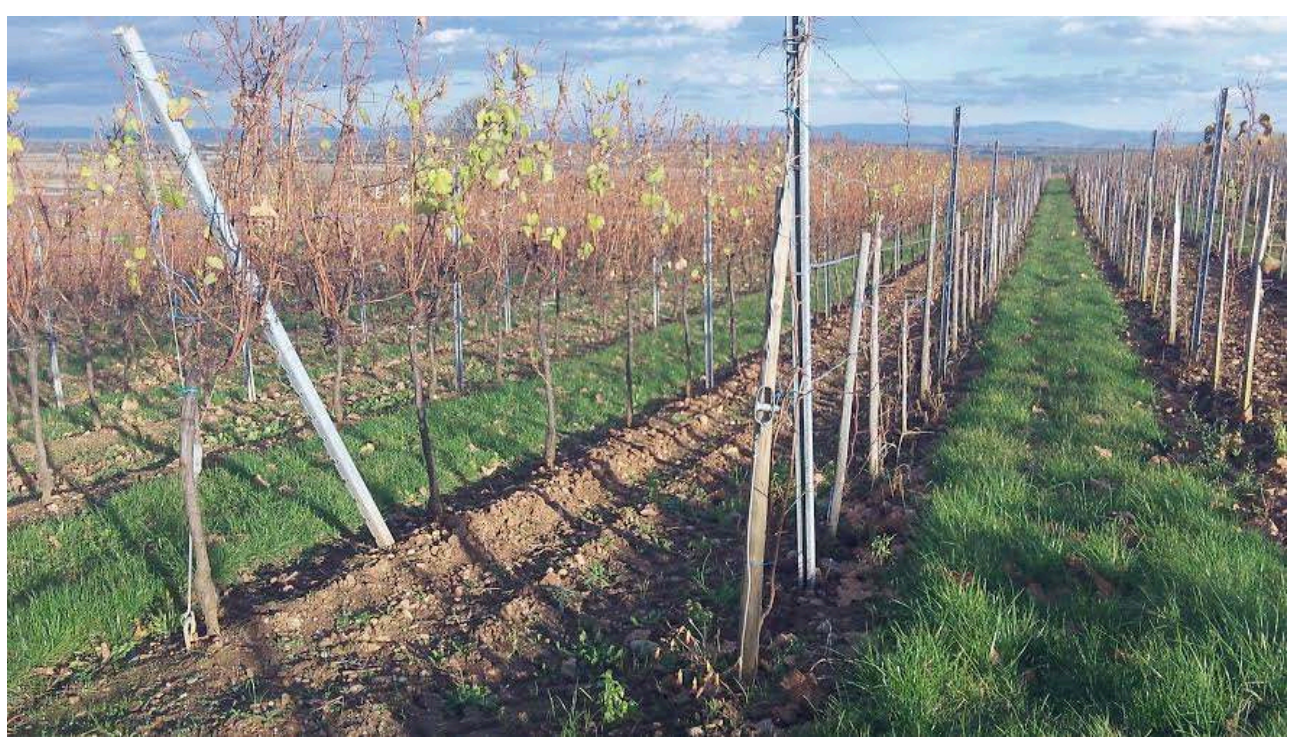

L'inter-rang laissé à nu est labouré pour faciliter l'infiltration des eaux et supprimer les adventices. Tous les 2 ans, les inter-rangs enherbés permutent avec ceux qui ne le sont pas. Beblenheim (HautRhin), novembre 2012, cliché : S. Boulanger

Parmi les vignerons adhérant à la Charte Vin Bio Alsace (des hommes à 94\%), 64,7\% ont repris une exploitation qui pratiquait de la viticulture conventionnelle. Plus de $29 \%$ desexploitants faisaient un autre métier avant de s'installer, lequel était dans $80 \%$ des cas lié au monde agricole : agronome, caviste, ingénieur agricole. La taille moyenne des exploitations est de 12,88 ha mais les écarts sont colossaux : de 0,5 ha à 40 ha et la comparaison des structures est difficile du fait qu'une part de ces exploitations est toujours en polyculture (vigne-maïs).

Les exploitations transmises ont été aussi bien créées au début des années 1970 qu'à l'aube des années 2000. Certaines continuaient donc de travailler en viticulture conventionnelle à cette date. Seules deux d'entre elles pratiquaient de la viticulture bio ou biodynamique. Nous relevons, à la suite des entretiens, que le fait de changer de système de production s'est imposé spontanément aux repreneurs, conscients que le système de leurs prédécesseurs avait peut-être atteint ses limites ${ }^{20}$ en n'étant plus en phase avec les attentes de la société en termes d'environnement. Pourtant, la conversion a engendré deux incompréhensions familiales parmi les personnes enquêtées : deux pères ne comprenant pas pourquoi leurs fils respectifs décidaient de passer de la viticulture conventionnelle, "produisant bien", à une viticulture aux moindres rendements et demandant plus d'efforts.

Les exploitants adhérant à Vinabio se répartissent selon trois tranches d'âges de façon presque équitable : un tiers appartient à la tranche des 30-40 ans, un autre à celle des 40-50 ans et le dernier, à celle des 50-60 ans. Aussi, élaborer du vin bio n'est ni l'apanage de la jeunesse, ni celui de l'âge plus mûr. Ce qui rassemble les exploitants réside dans leurs motivations à élaborer du vin bio : pour ces vignerons, la démarche est ressentie comme absolument nécessaire pour respecter la terre, les raisins, la santé du consommateur, celle de leur famille et la leur.

38 A la question "parmi vos pratiques viticoles, quelles sont celles qui, pour vous, symbolisent le plus votre engagement en faveur des vins issus de raisins 
biologiques?", presque $60 \%$ des vignerons interrogés évoquent d'abord le travail du sol puis le traitement par les plantes. Le travail du sol est parfois fait de façon attelée : cela évite le tassement lié aux roues du tracteur donc limite la formation d'une croûte imperméable et l'érosion. Le travail du sol revêt d'ailleurs une importance particulière dans ce vignoble de piémont aux parcelles parfois très accidentées. Le respect de la biodiversité, de l'écosystème et des sols, sont aussi des éléments récurrents du discours. Quelques réponses évoquent l'idée selon laquelle il faut accepter que les vins ne soient pas parfaits ou de qualité équivalente d'une année sur l'autre. Il s'agit d'un élément important car l'un des freins régulièrement évoqué par les vignerons en général à l'essor du vin bio réside dans la forte variation du goût et de la qualité du vin chaque année, alors que les acheteurs apprécient de déguster une qualité régulière. Pour mettre en place leurs pratiques viticoles, les vignerons ne font cependant guère appel à un technicien viticole extérieur (seuls 17\% d'entre eux le font). 53\% des vignerons lisent des revues spécialisées régulièrement, $6 \%$ n'en lisent jamais, et $11.7 \%$ rarement. L'empirisme et la transmission orale du savoir sont jugés comme étant plus essentiels.

Les réponses sur les pratiques viticoles témoignent de l'engagement des vignerons en faveur de la biodynamie pour plus de $50 \%$ d'entre eux ${ }^{21}$.La mise en place de celle-ci, jugée plus respectueuse encore de l'environnement et de la vigne, a été favorisée en Alsace par des facteurs d'ordre naturel : le climat estival sec et chaud, moins propice aux maladies qu'ailleurs rend les moyens prophylactiques moins nécessaires; les sols argileux sont plus favorables à l'enherbement que les sols granitiques. Mais les facteurs culturels sont déterminants dans l'essor de la biodynamie, telle la proximité avec l'Allemagne et la Suisse, pays précurseurs (et originel pour l'Allemagne).

Aussi motivés soient-ils dans leurs pratiques, ces vignerons cherchent-ils à faire des émules dans leur profession et leur région?

\section{Une démarche surtout personnelle, valorisante en terme d'image mais pas toujours spontanément affichée auprès des visiteurs}

41 Pour être réellement efficace en termes d'environnement la viniculture bio doit continuer de s'étendre. En effet, «les principaux problèmes auxquels sont confrontés ces agriculteurs (bio) proviennent des déséquilibres écologiques occasionnés par les pratiques agricoles de voisins n'ayant pas encore opté pour des formes de production plus respectueuses de l'environnement. L'agriculture bio ne pourra vraiment se développer et entraîner l'agriculture conventionnelle avec elle que le jour où elle aura atteint un seuil minimal (au moins 20\% des surfaces agricoles» (Dufumier, 2012). La condamnation d'un vigneron bourguignon bio ayant refusé de traiter ses vignes contre la flavescence dorée souligne le long chemin à parcourir pour convaincre la profession d'une extension du bio ${ }^{22}$. Les vignerons alsaciens ayant signé la Charte Vin Bio Alsace cherchent-ils à convaincre les exploitations voisines à suivre leur engagement? Seuls $23 \%$ d'entre eux l'affirment nettement, tandis que $50 \%$ ne le font pas, les vignerons qui restent disant le faire parfois tout en jugeant que cela ne relève pas de leur ressort. Ces réponses très éparpillées sont à relier avec l'idée selon laquelle l'engagement en faveur du bio est avant tout une démarche personnelle. En effet, les efforts liés à un surcroît de travail d'observation et le changement d'organisation de l'exploitation sont tels que pour s'engager dans la conversion biologique le vigneron doit être profondément motivé par sa démarche. Quatre vignerons ont par ailleurs affirmé avoir été convaincus 
de passer en mode biologique en regardant leur voisin pratiquer un changement de ses pratiques.

Les consommateurs jugent les produits biologiques encore trop chers, comme l'atteste l'étude de mai 2014 de l'Agence bio : 78\% des personnes interrogées estiment que le principal frein à l'achat de produits biologiques reste le prix (Agence Bio, 2014). Cet argument est régulièrement évoqué lors des visites au caveau mais le consommateur, lorsqu'il apprécie un vin après dégustation chez les vignerons alsaciens enquêtés, est toujours prêt à assumer un prix plus élevé car sa santé prime avant tout. Pourtant, lorsque le visiteur se rend au caveau, seuls $29 \%$ d'exploitants évoquent automatiquement leurs pratiques biologiques. Certains (59\% des exploitants) pensent aussi qu'il est nécessaire de valoriser leur production bio par une documentation et un discours spécifiques. Ils ont notamment édité des plaquettes où le paysage tient une place notoire, illustrant parfaitement l'affirmation de M. Duvigneau: «Le campagne aujourd'hui est un paysage avant d'être un lieu de production. Et si cette opinion est majoritaire pour les ruraux comme pour les urbains, elle est encore plus nette chez les urbains que chez les ruraux » (Duvigneau, 2002).

D'autres vignerons sont convaincus au contraire que leur démarche de production est naturelle et la seule viable à terme. Alors, pourquoi chercher à la valoriser d'une façon spécifique si elle doit tendre à devenir la norme?

\section{Conclusion}

Les pratiques de viticulture durable se sont indubitablement diffusées dans les vignobles français depuis les années 1980. Même si elles demeurent multiples et difficiles à classer, leurs points communs résident dans la méfiance et la moindre utilisation (voire l'absence d'utilisation) de produits de traitement de synthèse. La notion de qualité environnementale domine. Pourtant, les motivations des vignerons pour développer ces pratiques (durables et bio) sont la plupart du temps personnelles et complexes. Des vignerons n'adhérent pas au label bio mais pratiquent le bio pour garder leur liberté d'action. D'autres adhèrent mais ne l'affichent pas toujours car ils ne veulent pas «être associés à ces nouveaux bio qui n'ont de bio, selon eux, que l'étiquette " (Barrey, Teil, 2009). Cette hétérogénéité des pratiques et la diversité des organismes certificateurs, visibles en Alsace comme dans les autres vignobles français, entravent la diffusion de l'image positive que leurs pratiques pourraient engendrer.

L'évocation du terroir demeure un élément récurrent du discours des vignerons alsaciens : pour permettre au «terroir de mieux s'exprimer» et fournir aux vins un goût propre à chaque parcelle grâce aux choix d'encépagement et de pratiques culturales de chaque exploitant, il s'agit de limiter l'utilisation d'intrants, tant dans les pratiques culturales qu'œnologiques. 
Annexe 1 : Enquête sur les producteurs de vins issus de raisins biologiques en alsace

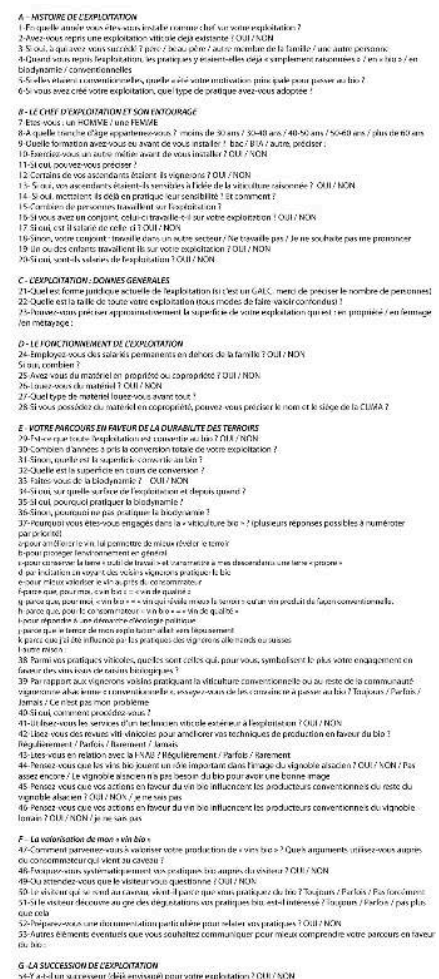

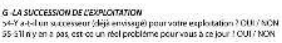

\section{BIBLIOGRAPHY}

Agence Bio, 2014, Baromètre de consommation et de perception des produits biologiques en France, $11^{\mathrm{è}}$ édition, $94 \mathrm{p}$.

Agence Bio, 2014, L'agriculture biologique, ses acteurs, ses produits, ses terroirs, Paris, La documentation française, $120 \mathrm{p}$.

Agreste 2010, Enquête sur les pratiques culturales des agriculteurs.

Agreste Primeur, septembre 2009, n²30.

Barrey S., Teil G., 2009, « La viticulture biologique : de la recherche d'un monde nouveau au renouvellement du goût de terroir ", Innovations Agronomiques, n4 , p. 427-440.

Dufumier M., 2012, Famine au sud, malbouffe au nord. Comment le bio peut nous sauver. SaintAmand-Montrond, Nil, 194 p..

Duvigneau M., 2002, Art, culture et territoires ruraux. Expériences et points de vue. Paris, Educagri, 322p.

Etude quinquennale 2010 sur la consommation de vin en France, France Agrimer, 27 novembre 2010. 
Fassier-Boulanger, 2008, « La viticulture durable, une démarche en faveur de la pérennisation des territoires viticoles français? », Géocarrefour, Vol. 83/3, p.181-190.

Fassier-Boulanger, 2006, « Paysages viticoles et évolution des pratiques culturales : les vignes hautes et larges et l'enherbement ", Sud-Ouest européen, $n^{\circ} 21$, p. 37-46,

Fischler C., Masson E., 2008, Manger. Français, Européens et Américains face à l'alimentation, Paris, Odile Jacob, 336 p.

Fischler C., 2000, « Alimentation contemporaine et perception du risque », Qu'est-ce que l'humain? Université de tous les savoirs, Paris, Odile Jacob, p. 239-248.

France Agrimer, 2014, Plan stratégique sur les perspectives de la filière vitivinicole à l'horizon $2025,32 \mathrm{p}$.

Legouy F., 2014, "La géohistoire de l'espace viticole français sur deux siècles (1808-2010) : plusieurs cycles viticoles décryptés.", EspacesTemps.net, Travaux, 17.02.2014.

Legouy F., 2008, « La France vitivinicole dans la mondialisation », Historiens et Géographes, p. 123-150.

Ministère de l'agriculture et de la pêche, 2008, Plan quinquennal de modernisation de la filière vitivinicole française, $19 \mathrm{p}$.

Peynaud E., Blouin J., 2012, Connaissance et travail du vin, Paris, Dunod, 370 p.

Poulain J.-P., 2008, «Les dimensions sociales et culturelles des enjeux de l'agriculture moderne », Les Entretiens Grandes cultures et société, 200 p.

\section{NOTES}

1. Commission des comptes de l'agriculture, 2013.

2. Le mildiou affecte les organes aériens de la vigne et provoque une diminution des rendements voire une perte des récoltes après un ralentissement de la maturation de la vigne.

3. Le rognage est l'élagage de la vigne en vue de favoriser la maturation du raisin.

4. Le phylloxera est un insecte importé des Etats-Unis qui s'attaque aux organes aériens et souterrains de la vigne. Il conduit à la ruine les vignobles français et européen à la fin du XIX ${ }^{\mathrm{e}}$ siècle et au tout début du XX⿳亠े siècle.

5. Les producteurs directs sont des vignes non greffées très sensibles au phylloxera.

6. Cette publication reprend les conclusions principales de l'enquête menée par sondage et réalisée conjointement par l'INRA et l'ONIVINS sur la consommation de vin par les Français. L'INRA et l'ONIVINS réalisent une enquête quinquennale depuis 1980 pour analyser les comportements individuels de consommation du vin (et des autres boissons) durant le repas et en dehors du repas.

7. Au même titre que l'ugni blanc en Charentes.

8. «Le développement durable est un développement qui répond aux besoins du présent sans compromettre la capacité des générations futures à répondre aux leurs ", rapport Brundtland, 1987.

9. Définition officielle du ministère de l'écologie, du développement durable et de l'énergie.

10. L'arrêté du 12 septembre 2006 a introduit plusieurs dispositions spécifiques à la gestion des effluents et défini précisément ce dernier terme..

11. Question écrite $n^{\circ} 19665$ de Roland Courteau, publiée dans le JO du Sénat du 06/10/2005, p. 2512. La réponse du ministre sera négative. 
12. La viticulture conventionnelle est fondée sur des traitements chimiques plus ou moins nocifs pour prévenir des maladies et des insectes nuisibles à la vigne. La viticulture raisonnée reste une viticulture conventionnelle.

13. Il s'agit du RCE 203/2012 qui est entré en application le $1^{\mathrm{er}}$ août 2012.

14. Toutes les données liées à l'agriculture biologique sont issues de l'Agence Bio.

15. L'objectif de $2012 \mathrm{du}$ Grenelle de l'environnement était d'atteindre $6 \%$ des surfaces.

16. Vinabio n'est pas la première charte sur la vinification des vins bio: Demeter, Biodyvin et Nature et Progrès existaient avant sa création. Mais Vinabio est la première élaborée au niveau d'une région viticole.

17. Ce n'est qu'en 2012 que l'UE crée le label vin biologique.

18. Sur 38 questionnaires envoyés, 26 ont été retournés. Les vignerons qui n'ont pas rempli le questionnaire, ni souhaité nous rencontrer pour le faire, ont principalement précisé qu'ils étaient trop accaparés ou ne jugeaient pas utile de justifier leurs pratiques. Le pourcentage proposé a pour finalité de faciliter la lecture des résultats.

19. L'enquête sur les pratiques culturales des viticulteurs en 2010 (Agreste) indique que $98 \% \mathrm{du}$ vignoble alsacien présentent un enherbement ce qui le place largement en tête des vignobles enherbés. L'enherbement est permanent pour $92 \%$ des surfaces viticoles soit plus 7 points par rapport à l'enquête de 2006. Il s'agit d'un couvert permanent, spontané pour les $3 / 4$ des surfaces enherbées, et semé pour le reste.

20. Pour les plus jeunes repreneurs, leur formation en BTA les a largement sensibilisés à la durabilité des pratiques agricoles contrairement aux retraités ou aux vendeurs.

21. La biodynamie, dont les principes ont été définis en 1924 par Rudolf Steiner, insiste sur le travail du sol (partie biologique) et la valorisation du sol et de la plante dans son environnement naturel grâce à des préparations issues de matières végétales, animales et minérales appliquées à des moments précis (partie dynamique).

22. Le viticulteur bio Emmanuel Giboulot a été condamné en avril 2014 à une amende de 500 euros pour avoir refusé de traiter ses vignes avec des produits chimiques contre la flavescence dorée.

\section{ABSTRACTS}

The French wine-producing industry has a big economic weight thanks to the to high-intensity agriculture of the 1950-1970's. Yet some this industry is experiencing economic difficulties since 1980's : pollution and environmental damage and desire for healthy food. So the winegrowers have gradually decided to develop some sustainable agricultural practices. The aim of this article is to explore how men try to put in place some sustainable wine producing in France particularly in Alsace.

Les bons résultats économiques de la filière viticole à l'international, issus de la révolution productiviste des Trente Glorieuses, ne doivent pas occulter les difficultés apparues à partir des années 1980-90: atteintes à l'environnement, demande sociétale en faveur de produits sains, volonté d'un "retour au terroir» dans le domaine alimentaire... Des formes de viticulture durable se développent donc, telle la viticulture raisonnée ou la viticulture biologique. Cet article se penche sur l'un des acteurs de la filière, le vigneron, afin de mettre en évidence les pratiques 
durables qu'il met en place, notamment dans le vignoble alsacien, vignoble précurseur dans ce domaine.

INDEX

Mots-clés: Alsace, terroir, viticulture biologique, viticulture durable, viticulture raisonnée Keywords: Alsace, integrated viticulture practices, organic production grapes, sustainable wine growing, terroir

\section{AUTHOR}

\section{SYLVAINE FASSIER-BOULANGER}

Paris-IV Sorbonne, laboratoire ENeC, UMR 8185, 191 rue Saint-Jacques 75005 Paris, sylvaine.boulanger@paris-sorbonne.fr 